\title{
Stereoscopic measurement of free surface flows
}

\author{
L. Chatellier ${ }^{\mathrm{a}}$, S. Jarny, F. Gibouin, and L. David \\ Institut P', CNRS - Université de Poitiers - ENSMA, UPR 3346 \\ Département Fluides, Thermique, Combustion \\ SP2MI - Téléport 2 \\ Boulevard Marie et Pierre Curie, BP 30179 \\ F86962 FUTUROSCOPE CHASSENEUIL Cedex
}

\begin{abstract}
The present paper adresses the problem of combined three-dimensionnal measurements of shape and velocity of moving free surfaces. A measurement method based on the cross-correlation of image pairs obtained from a calibrated stereoscopic vision system is presented. The underlying concept of the method consists in the generation of parametric shape and displacement forms which are directly projected on the camera models. This procedure is then integrated in an iterative optimization process so that elevation, orientation, curvature and displacement of each surface subset are accurately estimated. Application is made on inclined plane flows of complex non-Newtonian fluids as an alternative to conventionnal rheometric devices.
\end{abstract}

\section{Introduction}

Advanced two- and three-dimensional Digital Image Correlation (DIC) methods are widely used in the material and solid mechanics community with applications to shape and deformation measurements (see [8] for a review). In the fluid mechanics community, the Particle Image Velocimetry (PIV) technique and its derivatives have become unavoidable tools for the two-dimensionnal analysis of a wide spectra of flows, and three-dimensionnal applications are becoming more and more common. Despite the many practical differences between DIC and PIV methods, their fundamentals are identical, and a common application can be found in the study of free surface flows.

Most common 3D surfacic DIC and PIV methods extend the concept of optimized 2D correlation of image subsets [1] in the context of stereovision so that $3 \mathrm{D}$ coordinates as well as the three displacement components can be obtained after triangulation [5].

For free surface or interfacial fluid flows, the three-dimensionnal Interfacial PIV technique (3DIPIV) [18] derived from the Stereo PIV (SPIV) [14] and refined SPIV algorithms [11] have been succesfully applied to the simultaneous measurement of both free surface shape and velocities. Other PIV-based techniques have been adapted to the measurement of free surface using an extension of the synthetic Schlieren method [12]. Alternative, non correlation-based techniques have been applied to the measurement of both shape and velocity of free surface flows [2], or to the 3D tracking of particles near a free surface [10], while a number of developments mainly focus on the measurement of the sole free surface $[17,19,6]$.

In an effort to provide measurement solutions adapted to the analysis of free surface flows, a DIC/PIV method is proposed that identifies parametric free surface shapes and tracks their displacements and deformations using the direct projection of the physical scene onto a calibrated stereovision system. For validation purposes, the technique is applied to an inclined plane flow of a polyoxyde ethylene solution.

\footnotetext{
${ }^{a}$ e-mail: ludovic.chatellier@univ-poitiers.fr
} 


\section{Measurement method}

The proposed measurement technique belongs to the family of DIC/PIV methods and has been developped for hydrodynamic and rheological applications as an alternative to the traditional SPIV-based algorithms used in the fluid mechanics community. The initial formulation used to represent and identify the measured surfaces is based on an Eulerian representation in Cartesian co-ordinates of the height of a moving fluid column and its successive derivatives. Formally, such an approach can be compared to the developments proposed by Helm et. al[7].

\subsection{Procedure}

The measurement method is organized as follows:

- The fluid domain is represented by Cartesian coordinates $(x, y, z)$, where $(x, y)$ correspond to a fixed reference plane, typically the flat bed of a flume.

- Each measurement point consists initially in a set of physical 2D coordinates $\left(x_{0}, y_{0}\right)$ representing a position of interest in the reference plane, and for which the fluid height, shape and flow velocity are unknown.

- A square-base free surface subset surrounding $\left(x_{0}, y_{0}\right)$ is identified and parametrized by its height $h\left(x_{0}, y_{0}\right)$, orientation $\left.\partial h / \partial \alpha\right)_{\alpha \in(x, y)}$ and curvature $\left.\partial^{2} h / \partial \alpha \partial \beta\right)_{(\alpha, \beta) \in(x, y)^{2}}$

- The displacement $\left(D_{x}, D_{x}, D_{z}\right)$ and linear deformation $\left.\partial D_{\alpha} / \partial \beta\right)_{(\alpha, \beta) \in(x, y, z) \times(x, y)}$ of the surface subset are measured.

For the measurement of free surfaces, the method is based on the reconstruction of image patterns generated from a parametric shape predictor and projected onto the camera models. The projected image coordinates are then textured according to their position in the experimental images using bilinear interpolation. The candidate parametric shape is therefore represented by patterns of gray levels corresponding to the candidate $3 \mathrm{D}$ scene as viewed from each camera. Cross-correlation is applied between these patterns so that a cross-correlation coefficient, or a cross-correlation map, is obtained. Thus, the measurement can be validated if the shape predictor leads to a cross-correlation coefficient approaching one. The full procedure is integrated into an iterative optimization algorithm which generates the shape parameters and whose objective function is the cross-correlation coefficient itself or a combination of the cross-correlation coefficients obained from more than one pair of cameras.

The measurement of three-component surface velocities is performed once surface subsets have been parametrized. Then, a similar procedure including both the shape and displacement parameters is used, in which cross-correlation is computed for each pair of sequential images. Thus, the objective function of the optimization algorithm is the sum of the cross-correlation coefficients returned from each camera sequence.

\subsection{Formalism}

\section{Image interpolation}

Classicaly, each grayscale image recorded by camera \#i is represented by a discrete distribution of gray levels $\tilde{g}_{i}\left(\tilde{u}_{i}, \tilde{v}_{i}\right)$ recorded at pixel $\left(\tilde{u}_{i}, \tilde{v}_{i}\right)$, and which will be interpolated as a continuous bivariate function $g_{i}\left(u_{i}, v_{i}\right)$ of real-valued sensor coordinates $\left(u_{i}, v_{j}\right)$ using bilinear terms.

\section{Free-surface identification}

A shape parameter $p_{s}$ comprised of the height, orientation and curvature of the unknown free surface subset located above a measurement point $\left(x_{0}, y_{0}\right)$ is defined as:

$$
p_{s}=\left\{h, \partial h / \partial x, \partial h / \partial y, \partial^{2} h / \partial x \partial y, \partial h^{2} / \partial x^{2}, \partial^{2} h / \partial y^{2}\right\}
$$


so that, for a point $(x, y)=\left(x_{0}+\Delta x, y_{0}+\Delta y\right)$ located in a neighbourhood of $\left(x_{0}, y_{0}\right)$, the candidate free surface elevation is expressed as the second order bivariate Taylor development of $h$ in terms of $(\Delta x, \Delta y)$ :

$$
z=h+\frac{\partial h}{\partial x} \Delta x+\ldots+\frac{\partial^{2} h}{\partial y^{2}} \frac{\Delta y^{2}}{2}
$$

Given the calibration of camera \#i with a pinhole camera model of matrix $M_{i}$, the sensor coordinates are obtained from the physical coordinates $(x, y, z)$ using the usual projection formula:

$$
\left[\begin{array}{c}
s_{i} u_{i} \\
s_{i} v_{i} \\
s_{i}
\end{array}\right]=M_{i}\left[\begin{array}{c}
x \\
y \\
z \\
1
\end{array}\right]
$$

Thus, for a stereoscopic system comprised of two cameras (\#1 and \#2), the normalized cross-correlation coefficient corresponding to the free-surface candidate at point $\left(x_{0}, y_{0}\right)$ is defined as follows:

$$
C\left(p_{s}\right)=\frac{\sum_{\Omega\left(x_{0}, y_{0}\right)}\left(g_{1}\left(u_{1}, v_{1}\right)-\bar{g}_{1}\right)\left(g_{2}\left(u_{2}, v_{2}\right)-\bar{g}_{2}\right)}{\sqrt{\sum_{\Omega\left(x_{0}, y_{0}\right)}\left(g_{1}\left(u_{1}, v_{1}\right)-\bar{g}_{1}\right)^{2} \sum_{\Omega\left(x_{0}, y_{0}\right)}\left(g_{2}\left(u_{2}, v_{2}\right)-\bar{g}_{2}\right)^{2}}}
$$

where $\Omega\left(x_{0}, y_{0}\right)$ is a discrete square neighbourhood of the point $\left(x_{0}, y_{0}\right)$ defining a list of physical coordinates $(x, y)$ over which the grayscale functions values $g_{i}\left(u_{i}, v_{i}\right)$ are evaluated, averaged as $\bar{g}_{i}$, and cross-correlated.

\section{Displacement measurement}

In a similar manner, a displacement parameter $p_{d}$ is defined as follows:

$$
p_{d}=\left\{D_{x}, D_{y}, D_{z}, \partial D_{x} / \partial x, \partial D_{x} / \partial y, \partial D_{y} / \partial x, \partial D_{y} / \partial y, \partial D_{z} / \partial x, \partial D_{z} / \partial y\right\}
$$

The candidate displaced positions of the initial surface subset are computed from the initial positions defined in Equation 2 using an additional first order development of $(x, y, z)$ in terms of $(\Delta x, \Delta y)$ :

$$
\begin{aligned}
& x \quad x+D_{x}+\frac{\partial D_{x}}{\partial x} \Delta_{x}+\frac{\partial D_{x}}{\partial y} \Delta_{y} \\
& y \rightarrow y+D_{y}+\frac{\partial D_{y}}{\partial x} \Delta_{x}+\frac{\partial D_{y}}{\partial y} \Delta_{y} \\
& z \quad z+D_{z}+\frac{\partial D_{z}}{\partial x} \Delta_{x}+\frac{\partial D_{z}}{\partial y} \Delta_{y}
\end{aligned}
$$

The real valued sensor coordinates for the final images and the corresponding grayscale function values are then computed from these displaced positions using the calibation matrices.

Once an initial free-surface subset has been identified, the correlation coefficient between two successive images obtained by camera $\# i$ is defined for the validated shape parameter $p_{s}$ and the candidate displacement parameter $p_{d}$ as:

$$
C_{i}\left(p_{d} \mid p_{s}\right)=\frac{\sum_{\Omega\left(x_{0}, y_{0}\right)}\left(g_{i}^{0}\left(u_{i}^{0}, v_{i}^{0}\right)-\bar{g}_{i}^{0}\right)\left(g_{i}\left(u_{i}, v_{i}\right)-\bar{g}_{i}\right)}{\sqrt{\sum_{\Omega\left(x_{0}, y_{0}\right)}\left(g_{i}^{0}\left(u_{i}^{0}, v_{i}^{0}\right)-\bar{g}_{i}^{0}\right)^{2} \sum_{\Omega\left(x_{0}, y_{0}\right)}\left(g_{i}\left(u_{i}, v_{i}\right)-\bar{g}_{i}\right)^{2}}}
$$


where the superscript ${ }^{0}$ refers to constant values calculated using the fixed parameter $p_{s}$ in the initial image. The variables without superscript refer to the final image and are obtained using both $p_{s}$ and the candidate displacement parameter $p_{d}$ according to Equations 2 and 6.

For a binocular stereoscopic system, the sum of the correlation coefficients for the displacement parameter is then:

$$
C_{12}\left(p_{d} \mid p_{s}\right)=C_{1}\left(p_{d} \mid p_{s}\right)+C_{2}\left(p_{d} \mid p_{s}\right)
$$

which is therefore used as the objective function of the optimization algorithm. Once the displacement parameter has been validated, velocities $\left(V_{x}, V_{y}, V_{z}\right)$ are classically estimated using the displacements $\left(D_{x}, D_{y}, D_{z}\right)$ measured between two set of images divided by the time lag separating the two exposures.

\section{Optimization algorithm}

The Nelder and Mead simplex algorithm [13] is used for maximization of the correlation functions 2 and 6 against parameters $p_{s}$ and $p_{d}$, respectively. The optimization algorithm is initiated using a first approximation of $p_{s}$ or $p_{d}$ and stops when the simplex reaches a limit size.

Such a measurement method presents several advantages: first, it allows the search for any type of parametric shape, and not only the localization of matching areas between two or more images, so that, for example, surface orientation, curvature and velocity gradients can be directly obtained. Second, the projection of parametric shapes to generate image patterns allows measurements at user-chosen locations, whereas classic stereo-matching techniques need an interpolation of the triangulated result to measure the surface at specific points. Third, the projection of parametric shapes is not affected by the respective position and orientations of the cameras, which suppresses some issues associated to image deformation and the need to rectificate images [4,11].

Concerning the reconstruction of image subsets according to a shape parameter, two aspects can be discussed. For surface subsets that are well aligned with their projection line on the camera sensor, the interpolated grayscale information will show poor contrast, as the corresponding pixels will be confined in a thin strip of the sensor. On the other hand, an image reconstruction based on surface subsets allows to take account of the physical size of the surface markers for the definition of the measurement neighbourhood, so that their apparent size can be controlled and will be identical on each camera. This point may prove useful in pratice for particle markers, such as those used in PIV, as their number, size and appearence in the correlation window are crucial parameters [20].

Intrinsequely, this principle of measurement is not limited to stereoscopic system, as the optimization step can handle any combination of cross-correlated images patterns which can be generated from more than two cameras, whithout extending the number of optimization parameters.

\section{Experimental setup}

\subsection{Inclined plane channel}

The experimental device consists in an inclined rectangular channel realized in PMMA and designed to generate impulsively started free surface flows, such as encountered in the case of dam-break situations (Figure 1). The channel is $350 \mathrm{~mm}$ in width, $690 \mathrm{~mm}$ in length and $100 \mathrm{~mm}$ in height, with an open-end that ensures a free flow condition along its lower edge and a compartment closed by a sluice gate in its higher part, which is used as a fluid tank. An adjustable resting frame allows a precise inclination $\alpha$ of the channel, which was set to $10^{\circ}$ in the experiment.

\subsection{Material}

The polyoxyde ethylene solution is prepared from $300 \mathrm{~g}$ powder of polyoxyde ethylene powder poured into $2700 \mathrm{~g}$ of deionized water, leading to a mass concentration of $10 \%$. Then the mixture is let at rest 
for two weeks to ensure the complete diffusion of polymer chains into the water. Homogeneity of the fluid during the experiment is ensured by a manual mixing of the solution prior to the measurement process. The density of the fluid is measured using a picnometer and a precision weighing machine and is equal to $1200 \mathrm{~kg} / \mathrm{m}^{3}$.

\subsection{Imaging apparatus}

\section{Acquisition devices}

This flow is recorded using two Jai CV-M2 CCD Cameras equipped with 50mm f/22 Nikon lenses and linked to the R\&D VISION HIRIS real-time image acquisition software. The cameras are disposed in a classic stereoscopic arrangement, symetrically oriented at $\pm 30^{\circ}$ from the normal to the channel floor around the main flow direction, and elevated of approximatively $0.8 \mathrm{~m}$, as shown in Figure 1. Sheimpflug mounts are used to align the focal plane of each camera with the channel floor.

\section{Camera calibration}

Each camera of the binocular stereoscopic system is independently calibrated using the method of Faugeras and Toscani [3]. The calibration markers consist in 260 circular dots regularly distributed on a two-level Cartesian grid Lavision Typ\#20 ( $x-y$ steps of $15 \mathrm{~mm}$, $z$-levels of $10.5 \mathrm{~mm}$ and $12 \mathrm{~mm}$ ) which are automatically detected using specific in-house pattern recognition algorithms [15]. The calibration pattern conforms to the coordinate system depicted in Figure 1, so that camera calibration can be directly included in the surface identification algorithm. An additional calibration has been carried-out using a more refined $0.5 \mathrm{~mm}$-step Cartesian grid positioned at height $\mathrm{z}$ locations, in order to assess the linearity of the optical system over the whole depth of measurement. Optical distortion was found to be negligible, so that the sole Typ\#20 calibration pattern associated to the Faugeras-Toscani calibration method could be used satisfyingly.

\section{Seeding and illumination}

Similarly to most of the measurement methods using cross-correlation of images, speckle image patterns are used in order to enhance the correlation signal. For that purpose, light sub-millimetric dark organic markers are randomly spread upstream of the measurement region during the displacement, while the flow is illuminated by a centered $600 \mathrm{~W}$ light normally aligned with the channel floor. An average of $10^{4}$ particles is recorded in each image, and contrast between the lightly colored fluid and the darker markers was found sufficient to efficiently apply the cross-correlation procedures (Fig. 2). Despite the need to spread the markers over the flow during the experiment, this surface seeding technique has the advantage of preserving the rheological properties of the fluid, which would not necessarily be the case if volumic seeding was used.
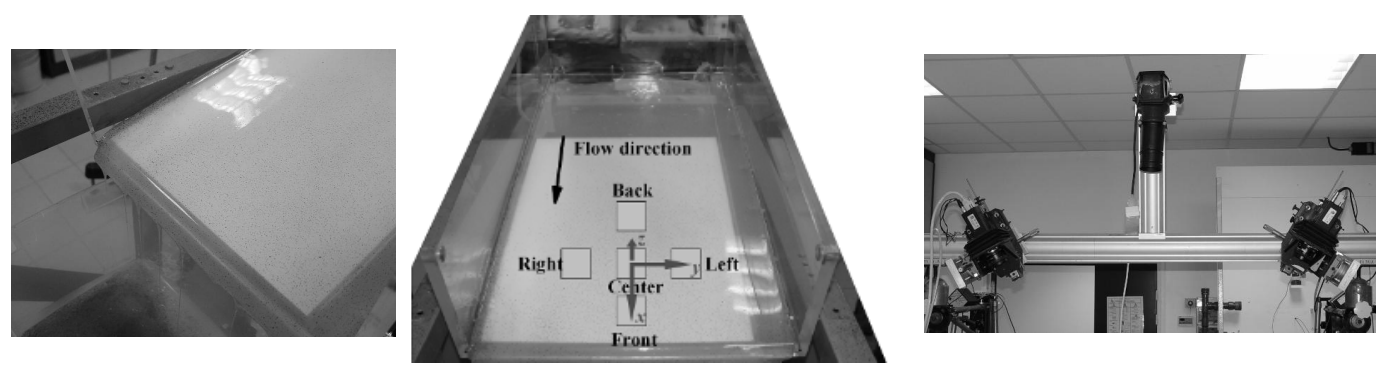

Fig. 1. Experimental apparatus and stereoscopic imaging setup. 


\section{Results}

A series of 500 double images of the polyoxyde ethylene flow is acquired at a rate of $1 \mathrm{~Hz}$, ensuring time-resolved measurements of the surface motion. The interrogation region is a $100 \mathrm{~mm} \times 120 \mathrm{~mm}$ cartesian grid situated at the free end of the channel and centered along the direction transverse to the flow. In order to assess the feasability of rheometric analyses using this experimental device, the interrogation region has been subdivided into the five $20 \mathrm{~mm}$ square zones depicted in Figure 1.

Synthetic particle images complying with the experimental devices have been analysed in order to assess the accuracy of the measurement. These preliminary results indicate that the free surface as well as millimetric displacements can be measured with a $50 \mu \mathrm{m}$ accuracy for slopes not exceeding $20 \%$, which is far beyond the actual measured values.

The identification of the surface shape and motion has been carried out on overlapping $12.5 \mathrm{~mm}$ square subsets over a $21 \times 25$ cartesian grid of $5 \mathrm{~mm}$ cell size. The experimental results show that the local free surface shape and local displacements are actually obtained with sub-millimetric accuracy, as it is observable in Figure 3, in which the disjoint surface subset and velocity distribution exhibit a good spatial continuity. According to the theory of inclined plane flows, in which only the streamwise component should be non-vanishing [9], a discrepancy criterion between this component and the norm of the velocity can be estimated. Here, the discrepancy is below $0.3 \%$ for the central zones and below $0.6 \%$ for the side zones, showing good agreement with theory.

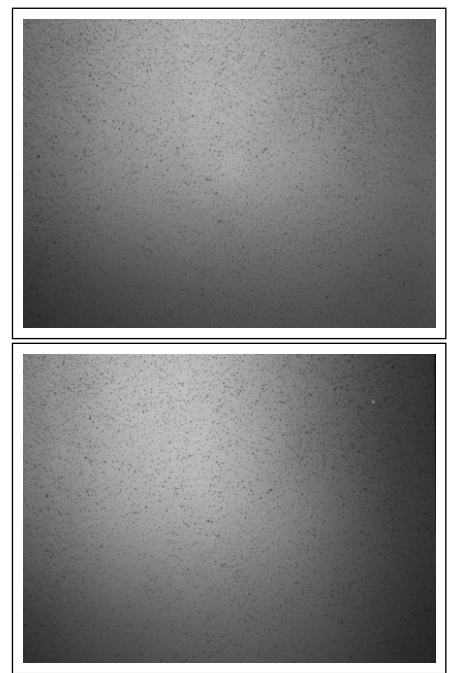

Fig. 2. Sample inclined plane flow image, as recorded from cameras Fig. 3. Sample result of surface and velocity measurements corresponding to \#1 (top) and \#2 (bottom).

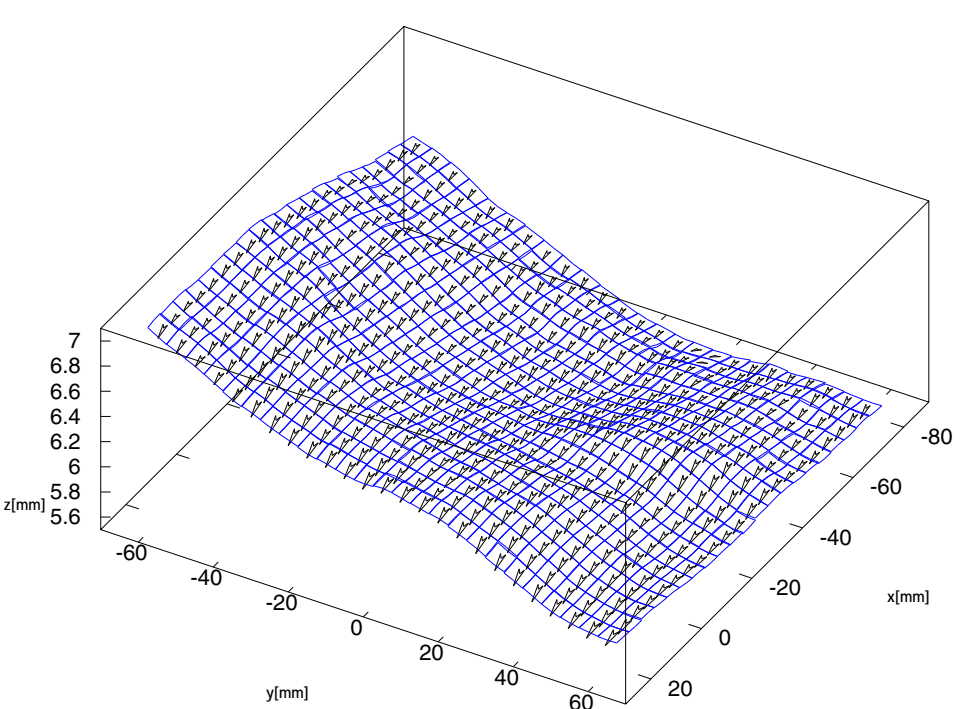
images of Figure 2. The average norm of the velocity vectors is $1 \mathrm{~mm} / \mathrm{s}$.

This series of data allows the extraction of height-velocity characteristics of the flow, leading to the identification of the rheometric law of the solution in terms of shear rate $\dot{\gamma}$ and shear stress $\tau$ at mid-height positions:

$$
\begin{aligned}
\tau & =\rho g \frac{h}{2} \sin \alpha \\
\dot{\gamma} & =\frac{V_{x}}{h}
\end{aligned}
$$

Figure 4 shows the rheograms resulting from the complete series of measurements, in which the free surface height decreases from $15 \mathrm{~mm}$ to $5 \mathrm{~mm}$ and the velocity from $1 \mathrm{~mm} / \mathrm{s}$ to 0 . These result can be compared to rheograms obtained over a wider range of shear rates with a classical rheometer using a coaxial cylinder geometry (Gemini, Malvern). 
The subdivision of the interrogation area into separate zones indicate that edge effects significantly affect the measurements in the left and right zones for high shear rates, showing that the uniform plane flow assumption can only be verified near the central line of the flume. The slight discrepancy of the rheograms obtained in the front, central and rear zones also indicate a slight increase of the shear rate near the free end of the flume.
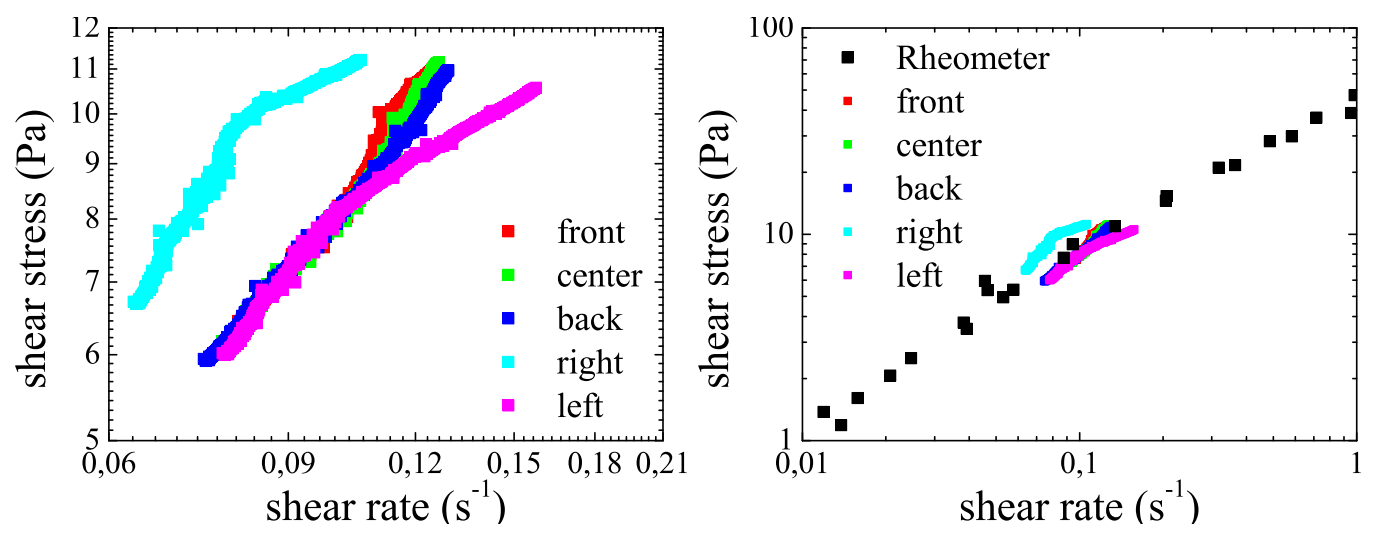

Fig. 4. Rheograms obtained from inclined plane measurements of the polyoxyde ethylene flow (left) and comparison with data obtained from a conventional rheometer (right).

\section{Conclusion}

A stereoscopic, cross-correlation-based, measurement technique has been developed to identify simultaneously the three-dimensional free surface and the three-component surface velocities of materials flowing down an inclined channel. The measurement method is based on the optimisation of parametric shape and displacement terms using an iterative algorithm. Tests based on synthetic images and experimental flows have led to statisfying results, showing a sub-millimetric accuracy of both shape and displacement measurements.

Rheograms from the inclined plane flow and a conventional rheometer have been succesfully compared and validate the principle of a rheometric application of this technique. However, the theoretical assumptions needed for the obtention of rheometric laws are hardly met over the whole channel, and slight discrepancies have been found to significantly alter the rheograms. An effort to enhance the experimental procedure and further assessment of the measurement accuracy is then needed.

This procedure has also been applied to clay suspensions [9] and seems to be an promising solution for the study of coarse suspensions, such as cohesive sediments, whose rheological characteristics are difficult to obtained using conventional rheometers.

\section{Aknowledgments}

The algorithms described in this paper have been implemented using the C++ Slip library (Simple Library for Image Processing) [16] jointly developped at XLIM and P', which the authors gratefully aknowledge.

This work is realized in a framework supported by CPER 13 and the FEDER program. 


\section{References}

1. H.A. Bruck, S.R. McNeill, M.A. Sutton, and W.H. Peters III. Digital image correlation using newton-raphson method of partial differential correction. Experimental Mechanics, 29(3):261267, 1989.

2. D. Douxchamps, D. Devriendt, H. Capart, C. Craeye, B. Macq, and Y. Zech. Stereoscopic and velocimetric reconstructions of the free surface topography of antidune flows. Experiments in Fluids, 39:533-551, 2005.

3. O. Faugeras and . Toscani. Camera calibration for $3 \mathrm{~d}$ computer vision. In International Workshop on Machine Vision and Machine Intelligence, Tokyo, Japan, pages 240-247, 1987.

4. A. Fusiello, E. Trucco, and A. Verri. A compact algorithm for rectification of stereo pairs. Machine Vision and Applications, 12:16-22, 2000.

5. D. Garcia, J.J. Orteu, and L. Penazzi. A combined temporal tracking and stereo-correlation technique for accurate measurement of $3 \mathrm{~d}$ displacements: application to sheet metal forming. Journal of Materials Processing Technology, 125-126:736-742, 2002.

6. B. Gautier and V. Valle. Mesure de reliefs en dynamique par moiré de projection couleur et analyse quasi-hétérodyne. In Proceeding of PhotomÃ C canique, Poitiers, pages 343-350, 2001.

7. J. Helm, S.R. McNeill, and M.A. Sutton. Improved three-dimensional image correlation for surface displacement measurement. Optical Engineering, 35(7):1911-1996, 1996.

8. F. Hild and S. Roux. Digital image correlation: from displacement measurement to identification of elastic properties : a review. Strain, 42(2):69-80, 2006.

9. S. Jarny, L. Chatellier, F. Gibouin, and P. Monnet. 3d surface and velocity measurements: application to inclined plane flows for rheological analys. In First European Congress of the IAHR, Edinburgh, UK, 2010.

10. M. Jehle and B. Jahne. A novel method for three-dimensional three-component analysis of flows close to free water surfaces. Experiments in Fluids, 44:469-480, 2008.

11. M. Jehle, S. Jarny, and L. David. Différentes approches pour la mesure d'interfaces et de surface libre. In 11ème Congrès Francophone de Techniques Lasers, Poitiers (France), pages 225-232, 2008.

12. F. Moisy, M. Rabaud, and K. Salsac. A synthetic schlieren method for the measurement of the topography of a liquid interface. Experiments in Fluids, 46(6):1021-1036, June 2009.

13. J.A. Nelder and R. Mead. A simplex method for function minimization. Computer Journal, 7:308-313, 1965.

14. A.K. Prasad. Stereoscopic particle image velocimetry. Experiments in Fluids, 29(2):103-116, 2000.

15. F. Scarano, L. David, M. Bsibsi, and D. Calluaud. S-piv comparative assessment: image dewarping+misalignment correction and pinhole+geometric back projection. Experiments in Fluids, 39(2):257-266, 2005.

16. B. Tremblais, L. David, D. Arrivault, J. Dombre, L. Chatellier, and L. Thomas. SLIP: Simple Library for Image Processing (Version 1.0). [Software]. University of Poitiers, France. http://www.sic.sp2mi.univ-poitiers.fr/slip/, 2010.

17. Ryota Tsubaki and Ichiro Fujita. Stereoscopic measurement of a fluctuating free surface with discontinuities. Meas. Sci. Technol., 16:1894, October 2005.

18. D.E . Turney, A. Anderer, and S. Banerjee. A method for three-dimensional interfacial particle image velocimetry (3d-ipiv) of an air-water interface. Meas. Sci. Technol., 20, 2009.

19. J. M. Wanek and C. H. Wu. Automated trinocular stereo imaging system for three-dimensional surface wave measurements. Ocean Engineering, 33(5-6):723-747, April 2006.

20. J. Westerweel. Fundamentals of digital particle image velocimetry. Meas. Sci. Technol., 8:13791392, 1997. 